\title{
Dictate a discharge summary
}

\author{
T M Penney
}

The main communication that a general practitioner receives about his or her patient's admission to hospital is the formal typed hospital discharge summary. This forms the core of available information and as such is of vital importance. Unfortunately, the task of dictating this summary is often regarded as a chore by the hospital doctor, which need not be the case if the job is tackled in a logical, systematic way.

At present there is a delay of about three weeks before the general practitioner receives the discharge summary..$^{1.3}$ The reasons for the delay are various but probably include delays in dictation and typing and postal delays.' If the summary can be dictated sooner, and in a way that requires the minimum of secretarial time, benefits will be reaped in several ways: the hospital doctor will enjoy up to date notes, the general practitioner will be aware of his or her patient's condition sooner, and the patients will be able to discuss their hospital stay and all its implications with their family doctor. Currently the system fails us.

I describe here how to dictate a discharge summary based on my own experiences and observations. I see no reason why most discharge summaries should not be received by the general practitioner within seven days of the patient's discharge if my guidelines are followed. With a standard format and better organisation the service can be vastly improved. It really is very easy to produce documents containing concise, relevant information if the right attitude to the task is adopted.

\section{Design}

I propose that all discharge summaries should be typed in one of two basic formats (figs 1 and 2). This would simplify the secretary's task and doctors would

Linden Avenue Medical

Centre, Kettering,

Northamptonshire

NN15 7NX

T M Penney, DRCOG, general practitioner
Date

Dr Smith
The Surgery
Anytown
Dear Dr Smith,

Re: Arthur BEST, 123 Anyroad, Anytown

Date of birth 1234

Hospital No 567890

Admitted

Discharged

Diagnosis

History

Past medical history

Medication on admission

Examination

Investigations

Treatment and progress

Medication on discharge

Follow up

\section{Yours sincerely, \\ Dr Jones, \\ Senior house officer to \\ Dr Brown, consultant}

FIG 1-Standard discharge summary

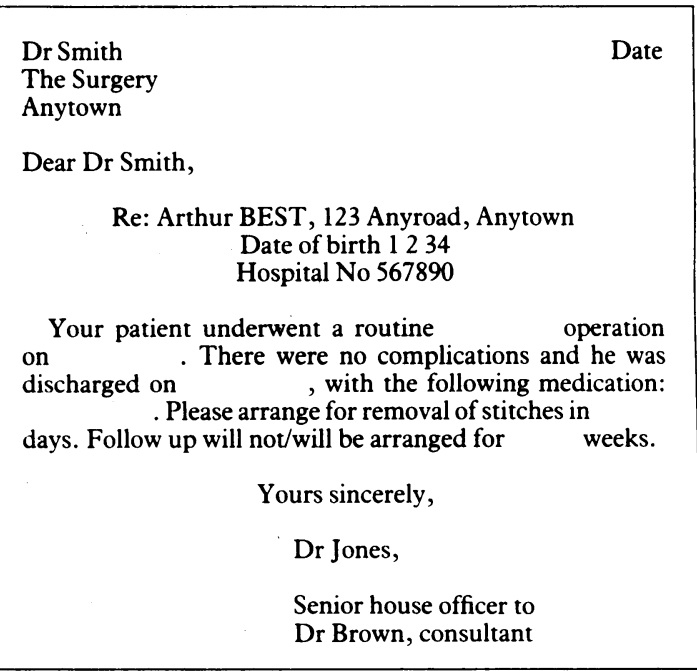

FIG 2-Short discharge letter

soon become accustomed to the design, so minimising the chance of any important details being omitted. Standard formats have been used successfully before. ${ }^{4}$

The short discharge letter may be used for any routine admission that is not complicated, when the general practitioner does not need detailed information. It lends itself especially to patients who have had operations and can be adapted to suit each particular admission. To stick rigidly to a detailed format for a routine appendicectomy, for example, wastes time and effort.

For most admissions the standard discharge summary can be used. Some of the sections, however, could be omitted in certain cases - for instance, "Past medical history," "Medication on admission," and perhaps even "Examination" and "Investigations." The general practitioner does not really need to know that "there were signs of consolidation at the right base posteriorly and a consolidated area in the chest radiograph" if the diagnosis of pneumonia of the right lower lobe is written at the top of the page.

\section{Details}

The details written under each heading in the discharge summary should be concise and relevant. Strictly speaking, only details relevant to the general practitioner to whom the letter is addressed should be included. The copy of the summary left in the hospital notes, however, can be extremely useful to a subsequent admitting doctor because it acts as a précis of past events. For this reason some hospital practitioners will prefer, for instance, to give negative results of investigations as well as positive ones in the summary. I contend that to keep summaries as short as possible, and therefore save as much time as possible, such details should be omitted. It takes only a few seconds to turn to the investigation sheets in the hospital notes. A short, relevant, and concise summary is much more likely to be read through by a busy general practitioner than a long letter full of irrelevant material.

The most important headings for the general practi- 
tioner are "Diagnosis," "Medication on discharge," and "Follow up." In cases in which a new diagnosis has been made a few details under an additional heading of "Information given to the patient" would be invaluable. Awkward situations have arisen when patients have not been given vital pieces of information that the general practitioner has taken for granted and misunderstandings have subsequently occurred.

When each senior house officer takes up a new post the consultant should go over the system of discharge summaries used in the department and give initial guidance on the standards expected.

\section{Dictation}

Now that a standard format has been designed and the information condensed time must be found to dictate the summary. In my experience no specific time is set aside for a senior house officer to dictate summaries. The time must be found during the course of an already busy day, and this is often after everyone else has gone home. I well remember spending hours sitting alone in a darkened office, occasionally interrupted by the cleaner. Many senior house officers take their work home with them, although this is not wise because of the problems that arise concerning the security and confidentiality of patients' notes once they are taken outside the hospital.

Time should be made available every week -30 minutes should be sufficient - to dictate summaries Perhaps the best time would be after the main ward round of the week, when decisions about discharging patients tend to be taken. Summaries could be dictated when the patients are sent home; the document will reach the general practitioner sooner, and the patients' individual details will be fresh in the mind of the senior house officer. Thus important details are less likely to be omitted and a better summary will be produced. If summaries are not dictated for several weeks it becomes more difficult to put a face to the patient's name. One has to thumb through notes to find the relevant information rather than being able to dictate most of the summary direct from memory.
I have experienced several disincentives to dictate summaries, the main one being a huge pile of notes overflowing from my pigeon hole in the consultant's office. A backlog develops surprisingly quickly, particularly at the start of a new hospital job. Often the senior house officer changes every six months, and the outgoing doctor may leave a residue of undictated notes behind. This is a dirty trick and quite unforgivable. The temptation would not be so great if dictation was performed promptly throughout the period of the job. Locums who do not dictate summaries on patients they look after present another problem; perhaps allocating the next senior member of the team to do the summaries while the senior house officer is away would help matters. A similar system could be adopted to cover holidays.

Equipment can also be a problem. Doctors should check that their dictaphones are recording properly and that their tapes are not damaged. I once came to the end of a long dictating session and checked the tape, only to find that the whole recording had been reduced to a series of unintelligible whirring and grinding noises.

\section{Dispatch}

After the summary has been dictated the tape should be labelled, attached to the notes, and left in a prearranged place for the secretary. Unfortunately, there is a general shortage of medical secretaries at present,${ }^{5}$ and typing may take several days or weeks. The senior house officer should make a habit of visiting the secretary's office regularly to sign the documents when they have been typed so that they may be posted without delay. Many hospitals use second class post.

1 Penney TM. Delayed communication between hospitals and general practitioners: where does the problem lie? Br Med I 1988;297:28-9.

2 Mageean RJ. Study of "discharge communications" from hospital. Br Med $\mathcal{J}$ 1986;293:1283-4.

3 Tulloch AJ, Fowler GH, McMullan JJ, Spence JM. Hospital discharge reports: content and design. Br Med $\mathcal{O}$ 1975;iv:443-6.

4 Stevenson JG, Murray Boyle C, Alexander WD. A new hospital discharge letter. Lancet 1973;i:928-31.

5 Miller HC. Delayed communication between hospitals and general practitioners. Br Med f 1988:297:292.

\section{ANY QUESTIONS}

Is a history of pulmonary embolus in the mother a contraindication to prescribing contraceptive pills to her daughters?

Although a family history of arterial disease is a relative (and sometimes an absolute) contraindication to the combined contraceptive pill, ${ }^{1}$ the same does not necessarily apply to venous thromboembolism. Pulmonary embolism is rarely familial, and the risk to the daughters is low, particularly if the mother's embolus had an obvious cause such as prolonged immobilisation. Nevertheless, there have been reports of families affected by recurrent venous thromboembolism, ${ }^{2}$ and the incidence of "familial thrombophilia" has been estimated at over one in 5000 . $^{3}$ It would, therefore, be wise to check the daughters for predisposing haematological abnormalities, ${ }^{3}$ such as thrombocythaemia or deficiency of antithrombin III, protein C, or protein S. If there are no such abnormalities and if the family is free of other diseases, such as ulcerative colitis, which may predispose to thromboembolism, the contraceptive pill may be prescribed. A pill containing less than $50 \mu \mathrm{g}$ oestrogen should be used, as this probably reduces the overall risk of thromboembolism. ${ }^{+}$- JAMES OWEN DRIFE, senior lecturer in obstetrics and gynaecology, Leicester

1 Guillebaud J. Combined oral contraceptive pills. In: London N, Newton J, eds. Handbook of family planning. Edinburgh: Churchill Livingstone, 1985.

2 Coon WW. Venous thromboembolism: prevalence, risk factors, and prevention. Clin Chest Med 1984;5:391-401

3 Mannucci PM, Tripodi A. Laboratory screening of inherited thrombotic syndromes. Throm Haemost 1987;57:247-51

4 Vessey M, Mant D, Smith A, Yeates D. Oral contraceptives and venous thromboembolism: findings in a large prospective study. Br Med f 1986;292:526.
Is there any evidence that anabolic steroids protect the bone marrow or promote its recovery from the ill effects of chemotherapy or radiotherapy?

No. Anabolic steroids do not protect the bone marrow, nor do they promote its recovery after cytotoxic treatment. There is no published evidence to support the idea, though agents like oxymetholone were probably used for this purpose in the 1970s. Oxymetholone stimulates the bone marrow through an increased production of erythropoietin and has been used previously to boost erythropoiesis in mild forms of hypoplastic anaemia. A major revolution is occurring in our knowledge of the regulation of haemopoiesis, and several growth factors have been purified, their genes have been cloned, and they are being produced by means of recombinant technology. Some of them, such as the colony stimulating factors GM-CSF and G-CSF (neutropoietin), are increasingly used to hasten the recovery of granulopoiesis after intensive chemotherapy and after bone marrow transplant procedures, thus reducing the period of neutropenia when the patient is at high risk of infection. Some of these factors, particularly G-CSF, have also been used to treat chronic neutropenia.' Recombinant human erythropoietin is specifically used to treat the anaemia of renal failure but has not been used extensively in cancer chemotherapy, although it has been suggested that it may have an effect on megakaryocytopoiesis (platelet production)

In summary, the modern trend is to use specific growth factors to stimulate haemopoiesis. It is not clear whether these factors act protectively during cytotoxic treatment, but the improvement in recovery after treatment is often spectacular and thus they may allow more intensive and effective regimens to be used safely in treating all forms of cancer.D CATOVSKY, professor of haematology, London

1 Jakubowski AA, Souza L, Kelly F, et al. Effects of human granulocyte colony-stimulating factor in a patient with neutropenia. $N$ Engl f Med 1989;320:38-42. 